\title{
Le roman de l'adultère : du mythe littéraire au palimpseste
}

\author{
Abdoulaye NDIAYE, Université Cheikh Anta Diop de Dakar, Sénégal
}

Dans sa configuration immédiate, le texte romanesque se dévoile imparfaitement à partir de sa seule mouture formelle. C'est l'analyse de ses différentes composantes qui en révèle des aspects non moins remarquables, comme celui consistant en sa rencontre avec d'autres textes souvent même éloignés dans le temps et l'espace. Le roman de l'adultère (celui du dix-neuvième siècle nous semble répondre le mieux à notre description) obéit à cette identité, en raison de sa «parenté génétique » avec le mythe littéraire, représenté par Le roman de Tristan et Iseut (douzième siècle). Le palimpseste apparaît alors comme le fil par lequel les deux textes dialoguent dans le temps et se révèlent dans toute leur plénitude par leurs formes et les histoires qu'ils charrient. L'aboutissement de cette interaction laisse voir que la civilisation doit passer par une grande révolution : le mariage dont dépend sa structure sociale est plus grave que l'amour qu'elle cultive et sollicite d'autres fondements qu'une «belle et intense fièvre ». L'analyse que nous en ferons prendra en compte la nature du texte premier et les relations qu'il entretient avec le texte de l'adultère du dix-neuvième siècle.

\section{I- La nature générale du mythe}

Devant la confusion des mœurs, le recours au mythe comme cause première autorise à se faire une idée exacte de l'ampleur de l'amour qui finit par la mort. Si le roman de Tristan est perçu comme un mythe, c'est en raison de la forte présence qu'il connaît chez les Occidentaux. C'est pourquoi une redéfinition du mot permettrait de comprendre qu'il est plus qu'illusion. Aristote emploie déjà ce terme dans la Poétique pour désigner l'intrigue, la structure narrative. Le mythe est histoire, donc narratif, contrairement au discours dialectique. Il est par essence irrationnel, intuitif contrairement à ce qui est purement philosophique. Le terme embrasse de nos jours tout un domaine sémantique allant de la religion aux beaux-arts en passant par la sociologie et la psychanalyse.

Dans son acception la plus large, le mythe est un récit d'auteurs anonymes qui met en exergue des questions d'origine et de destin. Il permet à l'homme de se positionner dans le temps, par rapport au passé et au futur, et il lui apporte assurance en proclamant son appartenance à une réalité continue, même si chaque mythe porte l'empreinte de la production d'une société définie, avec ses structures particulières, dont il garantit en retour la 
légitimité. Tout mythe est constitué d'un ensemble limité de motifs dont la particularité est qu'ils sont non littéraires et marquent quelques situations ou attitudes fondamentales. C'est au cours de l'élaboration secondaire que les motifs composant le mythe entrevoient le tissu d'un récit et «le thème particulier que cristallise et individualise le motif mythique est potentiellement un objet littéraire dans la mesure où il pourra générer un nombre infini de transpositions du modèle initial. »(Le Galliot 87). Ainsi posé, apparaît plus clairement la nature du mythe de l'adultère.

\section{II- Le mythe de l'amour-passion.}

Nous avons montré plus haut que le mythe désigne une fable, une histoire dont le signe pertinent est de signifier des situations à peu près identiques. Il permet, dans le flux des activités quotidiennes, de traduire un ensemble de faisceaux interdépendants. Plus concrètement, les mythes apportent au groupe social ou religieux les règles de conduite qui sont, à n'en pas douter, un élément fondamental pour sa survie. Cependant la particularité du mythe est son absence de paternité et son origine mystérieuse. Il est clair ainsi que roman et mythe sont absolument opposés. La valeur fondamentale du roman est la touche particulière de son auteur, l'esthétique de sa propre création. Mais le mythe se distingue plus radicalement du roman en ce que le premier exerce une fascination sur nous, que nous le voulions ou non. C'est ainsi qu'un évènement, une histoire finissent par devenir des mythes, alors que le roman est laissé à l'appréciation du public qui le juge et même le critique ; la configuration du mythe, elle, refuse la critique et « chasse » la raison.

Les lois contenues dans Le roman de Tristan et Iseut, perçues comme exemple des relations de l'homme et de la femme dans la société courtoise du douzième siècle, constituent l'archétype sur lequel se dessinent les lois de l'existence moderne, quoique celles- là soient bannies de manière officielle, mais leurs marques restent vivaces dans les esprits. La légende de Tristan recouvre trois traits qui l'assimilent à un mythe : l'auteur (s'il en existe) inconnu, l'élément sacré qu'utilise la légende (ici la coutume de la chevalerie médiévale) et la part de mystère qu'elle comporte et qui tient à un nœud; point de mythe sans la volonté d'obscurcir l'origine et la portée des faits symbolisés par le même mythe pour détourner l'attention du critique.

Son apparition est liée à une situation où toute impossibilité est acquise de révéler un certain nombre de faits sociaux ou de relations affectives que l'on veut cependant garder ou qui manifestent ostensiblement un droit à la pérennité. Ici, le fait mystérieux exprimé par le mythe est l'amour-passion lié à la mort. Une ambiguïté demeure cependant : une volonté de préservation de la passion, le souhait du malheur face aux morales officielles et à la raison 
qui se chargent de les fustiger. La nébuleuse du mythe offre ainsi l'opportunité d'appréhender le contenu flottant qu'elle laisse à l'imagination. Ainsi certaines réalités humaines senties comme fondamentales sont préservées de la critique. Le mythe a charge de dire ces réalités, parce que quelque chose en l'homme le demande, mais en y mettant des formes pour éviter le coup de semonce de la raison. En outre, depuis les études sur les mythes menées par ClaudeLevi Strauss (Anthropologie structurale),la notion de transhistoricité note la position par laquelle un dialogue entre des historicités distinctes et distantes met dos à dos, tout en les éclairant, des situations du passé et celles d'aujourd'hui .Dans le roman de l'adultère, «la représentation de la représentation classique » (voir Foucault) est une opération qui a recours à la notion freudienne de travail de deuil pour apporter plus de consistance à l'analyse du processus historique (du texte de référence $\mathrm{au}(\mathrm{x})$ texte(s) référant(s)). En conséquence, la transhistoricité doit nécessairement aboutir à une fécondation.

Le mythe de Tristan, stricto-sensu, se constitue au XIIe siècle, au moment où un vaste mouvement de mise en ordre sociale et morale s'opérait. L'enjeu était de mettre fin aux velléités destructrices de l'instinct qui réagissait aux attaques intempestives de la religion. Ce siècle vit apparaître une véritable crise du mariage. La réaction était imminente. Le Roman de Tristan et Iseut connut un succès dont la signification était principalement comprise dans la volonté d'ordonner la passion dans un cadre bien précis.

Pour prendre contact avec l'essentiel du récit mythique, nous empruntons la synthèse faite par Denis de Rougemont à partir de la concordance établie par Joseph Bédier entre les cinq versions du douzième siècle : Béroul, Thomas, Eilhart, La Folie Tristan et Le roman en prose. La naissance de Tristan coïncide avec la mort de ses parents, d'abord son père, ensuite sa mère Blanchefleur. Il n'est pas étonnant qu'il tire son nom de cette atmosphère de mort et de tristesse. C'est son oncle, le roi Marc de Cornouailles, frère de Blanchefleur, qui prend l'orphelin sous son aile protectrice et se charge de son instruction. Le jeune garçon commence par une série d'exploits dont le plus connu reste le combat qu'il mène contre le Morholt, un géant irlandais qui exige (à l'image du Minautore) un tribut constitué de jeunes filles et de jeunes gens de Cornouailles. Dès sa puberté, au moment d'être armé chevalier, il demande l'autorisation royale de le combattre; il y arrive, le tue mais reçoit un coup d'épée empoisonnée. Croyant sa mort imminente, il mène l'aventure dans un bateau sans voile ni rames, son épée et sa harpe comme compagnes. Son voyage le mène sur les bords irlandais. La reine d'Irlande Iseut, seule détentrice du secret du remède qui peut sauver Tristan, est la sœur du défunt grand Morholt. Tristan cache à la reine la cause de sa blessure, obtient qu'elle le soigne et finalement guérit. C'est le prologue. 
Plusieurs années ont passé. Le roi Marc s'engage à épouser la femme dont un oiseau lui apporterait un cheveu d'or. Tristan est désigné pour aller « à la quête » de l'inconnue. Une fois en Irlande, Tristan y mène un combat contre un dragon qui terrorisait la ville (...). Blessé de nouveau, Tristan est soigné par Iseut qui tarde à découvrir que le meurtrier de son oncle n'est autre que le blessé qu'elle soigne. A l'instant où elle se saisit de l'épée de Tristan, le jeune homme lui révèle la mission dont le roi Marc l'a chargé. Et Iseut lui fait grâce puisqu'elle veut devenir reine. Tristan et la princesse se dirigent vers les terres du roi Marc. Comme il fait chaud et qu'ils soif, la servante Brangien leur sert à boire. Par mégarde, ils boivent le « vin herbé » destiné aux époux et qu'avait préparé la mère d'Iseut. Dès cet instant ils entrent dans une destinée «qui jamais ne faudra de leurs vies, car ils ont beu leur destruction et leur mort» (De Rougement 22). Ils s'avouent ainsi leur amour et y cèdent. Même si la faute est consommée, Tristan se considère toujours comme le mandataire du roi et consent à conduire Iseut à Marc. Brangien, substituée à Iseut par ruse, passera la première nuit nuptiale avec le roi, sauvant ainsi sa maitresse du déshonneur, tout en expiant l'erreur fatale qu'elle a commise.

Cependant des barons «taupes» dénoncent au roi l'amour de Tristan et d'Iseut. Tristan est banni. Mais usant d'une nouvelle ruse (scène du verger), il convainc Marc de son innocence et est de nouveau repris à la cour. Le nain Frocine, complice des barons, tend un piège aux amants. Il sème de la «fleur de blé » entre le lit de Tristan et celui de la reine. Tristan, auréolé d'une nouvelle mission par le roi, cherche coûte que coûte à revoir sa bienaimée, à la veille de son départ. Pour rejoindre le lit de la reine, il fait un saut mais à cause du saut une ancienne blessure à la jambe se rouvre. Marc et les barons, informés par le nain, surgissent dans le dortoir .Ils remarquent des taches de sang sur la fleur de blé. La preuve de l'adultère est ainsi établie. Iseut sera ainsi punie : elle est «offerte » à des lépreux alors que Tristan est condamné à mort. Il s'évade (scène de la chapelle). Il délivre Iseut, et avec elle disparaît dans la forêt. Durant trois ans, ils y mènent une vie « aspre et dure ». Un jour, Marc les surprend endormis. Mais comme Tristan a eu l'idée de déposer entre leur corps son épée nue, le roi Marc, frappé par ce spectacle de chasteté, les épargne. Il ne les réveille pas, prend l'épée de Tristan et laisse sur place sa propre épée.

Les trois ans écoulés, le philtre ne fait plus d'effet. Tristan commence à se repentir, Iseut à regretter la cour. Ils consentent à aller trouver l'ermite Ogrin qui se chargera de remettre au roi sa femme. Marc promet son pardon. Les amants, à l'approche du cortège royal, se séparent. Iseut supplie Tristan de ne pas s'éloigner du pays au cas où le roi ne la traiterait pas bien. Ayant obtenu l'accord de Tristan, la reine déclare qu'elle n'hésitera pas à 
rejoindre le chevalier au premier signal de sa part, «ni tour, ni mur, ni fort chastel ». Chez Orri le forestier, ils se retrouvent souvent. Mais les barons félons veillent sur les sorties de la reine. Celle-ci demande et obtient un «jugement de Dieu» pour prouver son innocence. Grâce à cette ruse, elle obtient gain de cause : avant de s'emparer du fer rouge qui laisse intacte la main de l'innocent, elle jure n'avoir jamais succombé dans les bras d'un homme, hormis ceux du roi et de son manant qui vient de l'aider à descendre de sa barque. Le manant, c'est Tristan déguisé.

Mais le chevalier est entraîné au loin vers de nouvelles aventures. Il croit que la reine ne l'aime plus. Il consent à épouser, au-delà de la mer, «pour son nom et pour sa beauté », une autre Iseut, l'Iseut « aux blanches mains ». Et fort justement, Tristan la laissera vierge, car il regrette «Iseut la bloie». Enfin, blessé à mort, Tristan fait appel à la Reine de Cornouailles, la seule qui puisse encore le guérir. Elle vient, dans son vaisseau à voile blanche, signe d'espoir. Iseut aux blanches mains l'attendait aussi. En proie à une morbide jalousie, celle-ci s'en vient au lit de Tristan et lui annonce que la voile est noire. Tristan meurt. Iseut la blonde débarque à cet instant, monte au château, embrasse le corps de son amant, et meurt. ${ }^{1}$

Si nous proposons la version de Bédier (la plus attestée de toutes) c'est d'une part pour rendre compte des rapports que le mythe entretient avec le roman et d'autre part mettre l'accent sur l'importance de l'histoire: la chevalerie et la morale féodale (les deux lois entrant en jeu) sont utilisées par l'auteur pour les besoins de rebondissement du roman. Ce qui semble fort logique dans la mesure où il existe une sorte de loi tacitement établie entre le romancier et le lecteur : la volonté que le roman se déroule avec ses aspérités. Les obstacles extérieurs qui «gênent» l'amour de Tristan et d'Iseut ne sont autres que des artifices romanesques. C'est dans cette mouvance que le vrai sujet de l'œuvre se donne à voir, que la passion déroule son fil jusqu'au bout. Pourtant ces obstacles ne sont pas insurmontables, mais nos héros semblent vouloir adopter à plusieurs reprises la séparation : l'épée nue, le mariage de Tristan.

Le romancier invente-t-il ces artifices narratifs pour «torturer» le lecteur (au sens de plaisir du texte) ou tout simplement le dérouter? Dans tous les cas les souffrances des amants fondent l'intérêt et la saveur du roman tel que désirés.

\section{III-Mythe et roman : le palimpseste ?}

Gérard Genette propose cette définition du palimpseste : «Un palimpseste est, littéralement, un parchemin dont on a gratté la première inscription pur lui en substituer une 
autre, mais où cette opération n'a pas irrémédiablement effacé le texte primitif, en sorte qu'on peut y lire l'ancien sous le nouveau, comme par transparence » (Palimpseste, 178). Plus loin, Genette élargit sa définition et l'oriente vers le champ littéraire où le palimpseste montre (au sens figuré) qu'un texte peut toujours en cacher un autre, mais qu'il le dissimule rarement tout à fait, et qu'il se prête le plus souvent à une double lecture où se superposent, au moins un hypertexte et son hypotexte (169).

Pour notre analyse, le roman de l'adultère du dix-neuvième siècle et Le roman de Tristan et Iseut, constituent respectivement l'hypertexte et l'hypotexte à partir desquels s'élabore par transformation ou par imitation la dérivation qui rattache l'un à l'autre. Nous convenons avec Genette que toutes les œuvres sont hypertextuelles ; nous prenons également le parti de localiser, à partir de notre corpus, les échos (si faibles soient-ils) dans Le roman de Tristan et Iseut en relevant les divers motifs qui jalonnent les textes mythique et romanesque. En effet, une rapide analyse du Roman de Tristan et Iseut permet de déterminer un certain nombre de motifs qu'il est facile de repérer dans n'importe quel roman de l'adultère du dixneuvième siècle. Selon les formalistes russes et les analystes formels allemands, le motif (motive en anglais, motiv en allemand) désigne les « unités élémentaires d'intrigue » (Wellek et Warren 304).

La structure narrative du roman de l'adultère laisse apparaître des motifs récurrents qui s'accordent avec une série de séquences actionnelles du récit, lesquelles séquences reposent sur l'alternance de phases d'amélioration et de dégradation, d'équilibre et de déséquilibre successifs ; le récit révélant un enchaînement de séries enclavées sur le modèle ci-dessous où les fonctions de la série 2 constituent le processus de réalisation de la série 1 :

\section{Série 1}

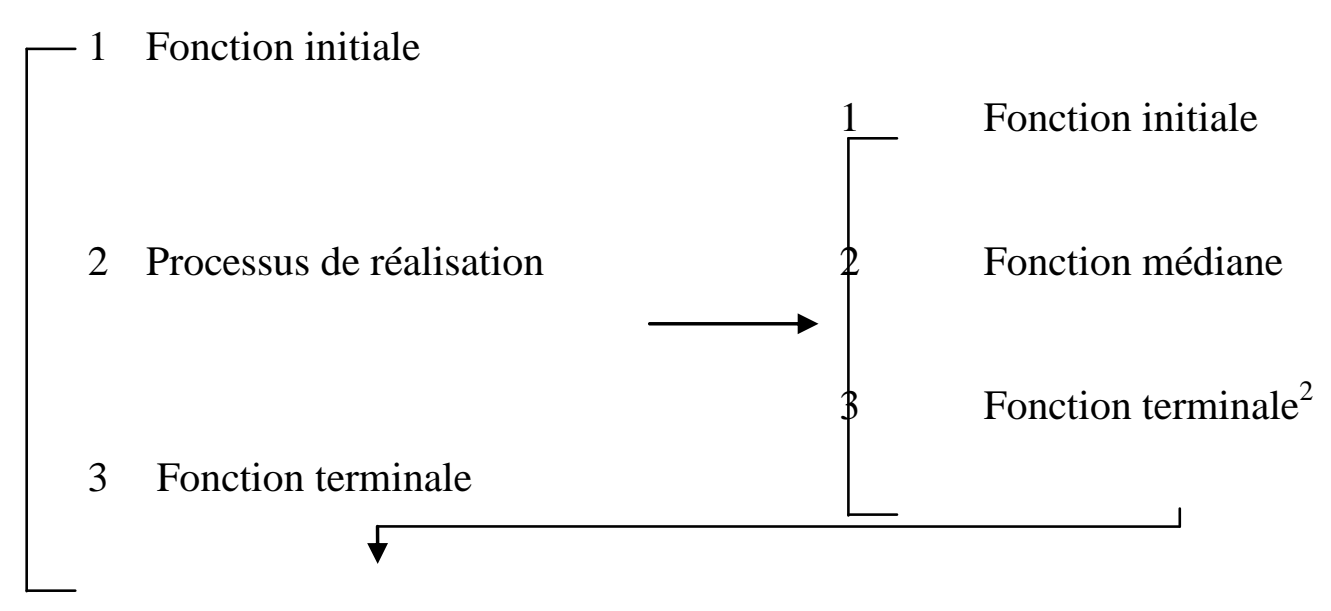


Il reste entendu que tout processus actionnel prend forme lorsque les trois moments ouvrant trois alternatives existent :

1. Virtualité : fonction qui ouvre ou non la possibilité d'un processus (conduite à tenir, évènement à prévoir) ;

2. Actualisation : fonction qui réalise ou non cette virtualité ;

3. Résultat : fonction qui clôt le processus : résultat atteint ou non. (Brémond 11-17) Si nous faisons appel aux triades et à la logique des possibles narratifs de Claude Brémond, c'est que ce dernier définit le récit moins comme une chaîne syntagmatique fixe de fonctions que comme un système narratif formé de bifurcations narratives et de l'oscillation constante du récit entre une série de directions possibles. La démarche de Brémond nous intéresse en ce qu'elle dépasse le postulat finaliste de Propp. Sa conclusion est sans ambages : « ne jamais poser une fonction sans poser en même temps la possibilité d'une option contradictoire. ${ }^{2}$

Cette logique des bifurcations possibles autorise à percevoir non seulement la linéarité mais mieux, un système de solidarité et d'enchaînement. Chaque moment coïncide avec un choix et, parmi des possibles, un seul devenir se trouve actualisé. La structuration du Roman de Tristan et Yseult recoupe aisément le schéma de Brémond qui pose les conditions d'une logique de l'action. Deux plans nous intéressant particulièrement ici (le contenu de l'histoire et sa mise en forme), nous essaierons de cerner les différentes étapes de l'histoire, les motifs rattachés à chacune d'elles et les fonctions mises en branle à cet effet).

La fonction initiale restitue le «motif précurseur »: le ménage constitué par le couple légitime (mari-époux) apparemment calme mais en réalité fort plat et ennuyeux, laisse place à la déception de la femme qui ne tarde pas à la concrétiser par un penchant à explorer un «ailleurs » plus adapté à ses pulsions intérieures. Si ce motif est clairement rendu dans le roman de l'adultère du dix-neuvième siècle, il l'est moins dans Le roman de Tristan et Iseut où la part de suggestion l'emporte sur l'explication. L'on devine cependant dans le mythe que ce qui unit les deux amants et les fait voir partout ensemble, c'est l'amour- passion qu'ils se vouent et la répugnance de la femme (qui, pourtant, a toujours rêvé de devenir reine) à vivre près de son mari. Dans tous les autres romans du corpus, la platitude du mariage constitue un motif omniprésent. C'est certainement dans la disposition formelle de ce motif où apparaissent les différences: Madame Bovary et Jean-Christophe respectent la structure linéaire chère au roman traditionnel où le déroulement chronologique suit une trajectoire prévisible: la platitude du mariage se révèle au fur et à mesure que les rencontres avec l'amant deviennent de plus en plus passionnantes. Ce motif se place dans la situation initiale 
et sert de passerelle pour le passage dans la situation où s'opère la transformation (épreuve qualifiante - épreuve principale - épreuve glorifiante).

Bel ami, La lettre écarlate et Une vieille maîtresse adoptent chacun un dispositif différent suivant l'option du romancier: Guy de Maupassant multiple et varie le procédé puisque son héros connaîtra plusieurs liaisons ; dans le roman américain, le lecteur se pose la question de savoir si le mariage de Hester Prynne et de Chillingworth est toujours effectif puisqu'aucun indice dans le récit ne lui donne l'opportunité de le croire, le mari et la femme se rencontrant en de rares occasions. On peut considérer tout de même que ce motif existe quoique de manière diffuse dans le roman de Nathaniel Hawthorne. Le cas d'Une vieille maîtresse est original par rapport aux autres romans puisque dans ce roman, l'on ne peut parler de platitude du mariage. Ryno de Marigny aime passionnément sa femme (qui le lui rend bien) mais l'intrusion d'un élément étranger dans ce couple jeune et amoureux crée le désordre dans les sentiments de l'homme et le pousse à tenter l'aventure (bien que cela lui pèse) avec cette maitresse si particulière. On parlera plutôt ici de déstabilisation du foyer opérée par Vellini. Cette fonction initiale traduit la virtualité dont nous avons parlé. C'est là où s'ouvre le récit et où commence à prendre forme l'adultère.

Le processus de réalisation qui a ses propres fonctions (initiale, médiane et terminale) offre au moins un motif commun au mythe et au roman. Si nous choisissons un motif aussi important que l'adultère en tant qu'action principale, nous constations qu'il est bien présent dans Le roman de Tristan et Iseut, il irrigue même toute l'œuvre. Le lecteur se posera certainement la question de savoir pourquoi Brangien qui est l'amie d'Iseut prend la place de cette dernière lorsqu'il ne lui restait plus qu'à s'offrir à son mari, le jour de son mariage. Il est facile alors de deviner que dans cette société du douzième siècle où les élites procédaient à un vaste effort de mise en ordre sociale et morale, il n'était pas recommandé à une jeune fille promise à un roi de perdre sa virginité. Et l'on comprend aussitôt que ce «manque »a une cause plutôt suggérée que rendue pleinement, l'ellipse étant passée par là. En dehors de $L a$ lettre écarlate qui porte plus sur l'après-adultère, tous les autres romans comportent ce motif récurrent qui prendra suivant les récits, des formes variables. Cette phase (processus de réalisation) correspond à l'actualisation de la virtualité qui s'était déjà manifestée.

Enfin la fonction terminale réalise la troisième alternative qui, comme on le sait, clôt tout processus actionnel du récit. C'est ici qu'apparaît le «motif clôturant » dont la forme la plus prisée par les romanciers, semble être la mort de l'homme ou de la femme adultère. Dans tous les cas, ce choix s'impose non seulement comme le " résultat » c'est-à-dire le troisième moment du processus actionnel (après la «virtualité » et l' «actualisation ») mais aussi 
comme un des « avertisseurs » dont dispose le romancier pour impulser une certaine morale à son œuvre. Il est intéressant de noter le cas que constitue La lettre écarlate dont l'originalité réside dans l'option du romancier : le roman débute là où finissent tous les autres. Mais il n'en demeure pas moins que le motif de la mort y constitue ce que nous avons souligné précédemment: sa forte présence sonne comme une invite à la réflexion morale. Dans $L e$ roman de Tristan et Iseut, il est facile de percevoir, à travers les péripéties mouvementées de l'histoire, les relents d'une terrible persécution qui s'abat sur le couple adultère et qui finit par provoquer la mort des amants. C'est cette même mort liée à l'amour qui explique la portée profonde du mythe sur la conscience occidentale, entre autres.

Il reste évident que la lecture du roman de l'adultère du dix-neuvième siècle renvoie inéluctablement au Roman de Tristan et Iseut en raison du contenu narratif commun à l'un et à l'autre. Nous venons de voir que la présence des motifs et d'autres non moins importants tels que les voyages, la jalousie, (l'éphémère) retraite mystique, le remords, etc. et les différentes articulations narratives spécifiques à chaque récit, offrent un vaste observatoire pour procéder à l'analyse du palimpseste. Nous y sommes poussé par la remarque de Michel Riffaterre sur l'intertextualité qui, dit-il, « est le mécanisme propre à la lecture littéraire. Elle seule, en effet produit la signifiance, alors que la lecture linéaire, commune aux textes littéraires et non littéraires, ne produit que le sens »(30). Nous considérons ainsi avec Riffaterre, que la «trace » intertextuelle va au-delà du seuil de l'ordre de la figure ponctuelle (du détail) et prend en compte l'œuvre vue dans la perspective de sa structure d'ensemble. ${ }^{3}$ C'est pourquoi mettre au clair la transcendance textuelle du texte est pour Genette, « tout ce qui le met en relation, manifeste ou secrète avec d'autres textes ${ }^{4}$.

Il est une autre opération (plus complexe, celle- là) qui consiste à déterminer comment le palimpseste se manifeste à partir du roman du dix-neuvième siècle. Nous nous contenterons de montrer que la transtextualité comme objet de poétique, dépasse et intègre l'architextualité et quelques autres types de relations transtextuelles qui sont au nombre de cinq (recensement de Genette) :

- l'intertextualité, analysée par Julia Kristeva, que Genette définit comme « la relation de coprésence entre deux ou plusieurs textes, c'est-à-dire eidétiquement et le plus souvent, par la présence effective d'un texte dans un autre » (Palimpsestes, 10).

- relation texte-paratexte qui est un champ de relations où la dimension pragmatique du roman, c'est-à-dire son action sur le lecteur, est le plus visible.

- Le troisième type de transcendance textuelle (ou métatextualité) est défini par Genette comme « une relation de «commentaire» qui unit un texte à un autre texte dont il 
parle, sans nécessairement le citer (le convoquer), voire à la limite, sans le nommer » (10). il donne l'exemple de Hegel dans_Phénoménologie de l'esprit qui évoque discrètement Le neveu de Rameau.

- Le quatrième type, l'hypertextualité, est pour Genette «toute relation unissant un texte B (hypertexte) à un texte antérieur A (hypotexte) sur lequel il se greffe d'une manière qui n'est pas celle du commentaire... » (11-12). C'est là où s'opèrent deux sortes de transformations : une transformation simple ou directe (elle consiste par exemple à transposer l'action de telle œuvre lointaine dans un lieu du dix-neuvième siècle) et une transformation plus complexe, plus indirecte; Genette cite le cas de L'Odysée et L'Enéide où Virgile raconte une toute autre histoire (les aventures d'Enée, et non plus d'Ulysse). Il s'inspire alors du type (générique) ${ }^{5}$ établi par Homère dans L'Odyssée.

Il se révèle que la notion d'hypertextualité s'amplifie et pourrait être améliorée, ce que fait Genette : il appelle désormais hypertexte «tout texte dérivé d'un texte antérieur par transformation simple (nous dirons désormais transformation tout court) ou par transformation indirecte: nous dirons imitation »(13). C'est là où il faut intégrer le cinquième type avec de nouvelles notions : le pastiche et la parodie. Genette en donne un aperçu :

C'est que, contrairement à la parodie, dont la fonction est de détourner la lettre d'un texte, et qui se donne donc pur contrainte compensatoire de le respecter au plus près, le pastiche, dont la fonction est d'imiter la lettre, met son point d'honneur à lui devoir littéralement le moins possible. La citation brute, ou emprunt, n’y a point sa place (84).

La part d'ironie qui couvre le roman du corpus plaide en faveur de la parodie alors que la volonté pour le roman de dix-neuvième siècle de se départir autant que possible des réalités du dix-neuvième siècle (bien que roman et mythe portent sur le même thème) le rapproche plus du pastiche. La première perception d'un lecteur averti qui parcourt Madame Bovary est incontestablement l'ironie qui traverse le roman, elle s'impose d'autant que tout le roman de Gustave Flaubert est bâti sur elle, elle constitue le socle à partir duquel l'histoire vient se greffer d'abord et se constituer ensuite. Toute l'originalité de l'œuvre repose sur un effet de présence et de défaillance à la fois du réel, ce que Jacques Neefs a bien analysé : «Flaubert maintient le «monde » de référence dans un mutisme qui le fait être l'implicite et l'opaque négatif de l'œuvre. Détails documentaires, indices sociaux et culturels, système des valeurs ne sont qu'appui défaillant, ensemble de réalités rendues dérisoire par l'effet même du sens qui les dispose toujours en creux de son apparition » (40). 
La grande dérision qui inonde Madame Bovary apparait mieux au vu des personnages Charles, Homais, Bournisien, s'il est admis que chez Emma et Charles par exemple, la naïveté est excusable et présente même des profondeurs qui laissent place (au niveau de leur conscience) à une dimension qui leur échappe, chez Homais par contre, aucun prolongement n'est possible. Il est l'incarnation de la médiocrité même. Comme un roman qui choisit de faire de tels personnages, les moteurs de tout évènement ou action, pourrait-il échapper à l'implacable tyrannie de l'ironie ? Mais cela lui donne-t-il droit à être considéré dans son entièreté, comme une pure parodie ? Tous les romans du corpus obéissent à la ligne qui consiste à donner de la représentation de l'adultère une teinte moralisatrice tout en veillant à respecter les convenances du pastiche. Si l'effet parodique ne nous semble pas primordial bien que présent, les raisons peuvent être cherchées dans le refus du roman du dix-neuvième siècle de respecter les normes et la structure de mythe. La souplesse du roman de l'adultère se manifeste davantage dans la part qu'il fait à la dimension tragique et à celle (plus esthétique, plus réaliste ?) de l'ironie.

En définitive, parodie et pastiche se partagent les contours du palimpseste perçu entre le roman de l'adultère et Le roman de Tristan et Iseut. Une vieille maîtresse et JeanChristophe s'offrent même le luxe d'intégrer un pan narratif du mythe dans le cours du récit. Quand, dans le roman de Romain Rolland, l'amant revient dans la demeure de sa maîtresse et que cette dernière, malgré la résolution qu'elle avait prise de ne plus le revoir jamais, va à se rencontre, le lecteur découvre brusquement l'analogie des situations ainsi juxtaposées :

Elle était près d'entrer dans la chambre de Christophe, quand elle sentit sous ses pieds nus au lieu du contact habituel du parquet lisse et froid, une poussière tiède qui s'écrasait mollement. Elle se baissa, toucha avec les mains, et comprit : une mince couche de cendres fines avait été répandue dans toute la largeur du couloir, sur un espace de deux à trois mètres. C'était Bâbi qui avait, sans le savoir, retrouvé la vieille ruse employée, au temps des lais bretons, par le nain Frocin pour surprendre Tristan se rendant au lit d'Yseult. Tant il est vrai qu'un nombre restreint de types, dans le bien comme dans le mal, servent pour tous les siècles. Grande preuve en faveur de la sage économie de l'univers ! (292)

De même Une vieille maîtresse adopte la même technique d'intégration du morceau ancien sur le nouveau. Il existe une séquence narrative commune au Roman de Tristan et Iseut et au roman de Jules Barbey D'Aurevilly où la différence ne se manifeste qu'à la fin du récit : dans le premier, le couple adultère meurt alors que dans l'autre il poursuit sa liaison. Il s'agit précisément de l'existence de deux Iseut dans le mythe et de Vellini et Hermangarde dans le roman. Le rapprochement est vite fait dans le roman de Barbey: Revenu auprès 
d'Hermangarde, l'Yseult aux blanches mains, toujours sereine comme la Résignation et d'une beauté inaltérée comme l'eau des sources, il (c'est-à-dire Ryno) avait mieux apprécié la différence qu'il y avait entre ces deux femmes (510).

On le voit, une lecture intertextuelle des romans de l'adultère du dix-neuvième siècle permet de confirmer la vue de Bakhtine et Kristeva sur la question : dans la littérature ce n'est pas seulement un sujet qui s'exprime mais une relation avec l'autre; écrivant, il se souvient de la lecture d'un autre.

\section{Conclusion}

Dans tous les cas, il apparaît, au vu du rapprochement établi entre le mythe de l'adultère et le texte romanesque, un ensemble de faisceaux interdépendants qui contribuent à éclairer la réalité intertextuelle. On conviendra avec Genette que l'art de faire du neuf avec du vieux présente l'intérêt de produire des «objets plus complexes et plus savoureux que les produits «faits exprès » : une fonction nouvelle se superpose et s'enchevêtre à une structure ancienne, et la dissonance entre ces deux éléments coprésents donne sa saveur à l'ensemble » (451). Nous avons montré en passant que pastiche et parodie « désignent la littérature comme palimpseste » (451) et la lecture relationnelle ouvre la voie à l'exploration de deux structuralismes : c'est, d'une part, celui de la clôture du texte et du déchiffrement des structures internes et d'autre part celui où il est facile de voir comment un texte (ici un mythe) peut en lire un autre. Nous n'avons pas analysé le mythe dans une perspective simplement historique mais aussi pour faire voir qu'il est une forme avec ses deux versants, statique et externe (la forme des objets; le volume des objets), dynamique et interne (la configuration née d'un mouvement). C'est toute cette dimension interne et externe à la configuration des textes qui leur assure toute leur littérarité et donne pleine mesure à la relation, à la limite «consanguine » entre le texte de référence (mythe) et le texte référant (le roman de l'adultère). L'intertextualité, dans ces conditions, s'entrevoit dans une sorte d'exigence de la reprise, de la redondance, de la redite des œuvres et qu'éclaire leur retour incessant dans l'histoire littéraire.

\section{Bibliographie}

Barbey d'Aurevilly, Jules. Une vieille maîtresse. Paris : Pléiade, 1974 (1851).

Bloom, Harold. The Anxiety of Influence. Oxford : Oxford UP, 1973.

Brémond, Claude. Logique du récit. Paris : Seuil, 1973.

Flaubert, Gustave. Madame Bovary. Paris : Librairie générale française, 1983 (1857). 
Fontane, Theodor. Effi Briest. Paris : Laffont, 1981 (1895).

Freud, Sigmund. L'interprétation du rêve. Paris : PUF, 2005.

Genette, Gérard. Palimpseste, la littérature au second degré. Paris : Seuil, 1982.

---. Introduction à l'architexte. Paris : Seuil, 1979.

Hawthorne, Nathaniel. La lettre écarlate. Paris : Belles-Lettres, 1955 (1850).

Hennique, Léon. L'accident de M. Hébert. Paris : Charpentier, 1884.

Kristeva, Julia. Séméiotiké. Paris : Seuil, 1969.

Le Galliot, Jean. Psychanalyse et langages littéraires. Paris : Nathan, 1977.

Lévi-Strauss, Claude. Anthropologie structurale. Paris : Plon, 1958.

Maupassant, Guy de. Bel-Ami. Paris : Grands textes classiques, 1994 (1805).

Neefs, Jacques. Madame Bovary. Paris : Hachette-Poche critique, 1966.

Riffaterre, Michael. «La trace de l'intertexte». La Pensée (octobre 1980).

Rolland, Romain. Jean- Christophe. Paris : Pléiade, 1973 (1902).

Rougemont, Denis de. L'amour et l'Occident. Paris : Union Générale d'Editeurs, 1960.

Tolstoï, Léon. Anna Karénine. Paris : Stock, 1972 (1870).

Wellek, René et Austin Warren. La théorie littéraire. Paris : Seuil, 1971.

\section{NOTES}

1- Résumé effectué à partir du récit de Denis de Rougemont, L'amour et l'occident, 21-23.

2- P. 11-17. Pour Brémond, l'unité de base, l'atome narratif, demeure la fonction, appliquée aux actions et aux évènements qui, groupés en séquences, engendrent un récit.

3- Harold Bloom a mené des recherches sur les mécanismes de l'influence. Cf Bloom, The Anxiety of Influence, Oxford UP, 1973.

4- Gérard Genette définit l'architextualité du texte ou architexte (ou encore la littérarité de la littérature) comme « un ensemble de catégories générales, ou transcendantes - types de discours, modes d'énonciation, genres littéraires, etc. - dont relève chaque texte singulier. »Introduction à l'architexte. Seuil : Paris, 1979.

5- Formel et thématique, ajoute Genette. 